\title{
Ligamentum Flavum Hematoma Associated with L5-S1 Pars Interarticularis Defect: A Case Report and Review of the Literature
}

\author{
Kenan Kıbıcl, ${ }^{1, \odot}$ Berrin Erok ${ }^{2, \odot ~ A h m e t ~ C ̧ o l a k ~}{ }^{3}$ \\ ${ }^{1}$ Department of Neurosurgery, Altınbas University School of \\ Medicine Bahcelievler Medical Park Hospital, İstanbul, Turkey \\ 2Department of Radiology, Cihanbeyli State Hospital, Konya, Turkey \\ ${ }^{3}$ Department of Neurosurgery, Pendik Medical Park Hospital, \\ İstanbul, Turkey \\ Indian J Neurosurg 2021;1:85-88.
}

\begin{abstract}
Address for correspondence Berrin Erok, MD, Department of Radiology, Cihanbeyli State Hospital, Cihanbeyli, Konya, Turkey (e-mail: drberrinerok@hotmail.com).
\end{abstract}

\begin{abstract}
Keywords

- ligamentum flavum hematoma

- radiculopathy

- pars interarticularis defect

- spinal instability

Ligamentum flavum hematoma (LFH) is an extremely rare compressive epidural lesion of the spine. The clinical manifestations of LFH are similar to that caused by other more common pathologies of nerve root compression like disc herniation. In the diagnosis, magnetic resonance imaging (MRI) is very important but challenging due to the changing intensities of the hematoma in relation to the stages of the aging blood. Herein, we report a case of LFH compressing the spinal canal in a 60-year-old man with pars interarticularis defect. He presented with low back pain radiating to the left leg. The neurological examination was consistent with left $\mathrm{S} 1$ nerve root compression. The MRI revealed a left posterior epidural mass compressing the thecal sac and S1 nerve root at the left L5-S1 level near the pars interarticularis defect. After surgical removal of the lesion, histopathological examination confirmed the diagnosis of LFH. Following surgery, he recovered rapidly. LFH due to spinal instability is important in terms of the understanding of the pathophysiological mechanisms related with LFH.
\end{abstract}

\section{Introduction}

Ligamentum flavum (LF) is the longest ligamentous structure in the human body. Its main function is maintaining the upright position. Different pathological changes can occur in LF including hypertrophy, calcification/ossification, and cyst formation. In addition, rarely hematoma may occur in the LF and it is an extremely rare cause of nerve root and spinal cord compression. The first case of LF hematoma (LFH) in the literature was reported by Sweasey et al in 1992. ${ }^{1}$ Although LF is extended from the $\mathrm{C} 2$ vertebra to the sacrum, the majority of reported cases of LFH are located at the mobile segments of spine, most frequently at lumbar region. ${ }^{2}$ The cervical and lumbar spine receives transmitted pressure to the epidural space due to their spinal mobility. Therefore, most intraligamentous hemorrhages are observed in the mobile cervical and lumbar regions. In the literature, the reported cases are

DOI https://doi.org/ $10.1055 / \mathrm{s}-0040-1713054$ ISSN 2277-954X. older aged, most frequently older than 60 years old, and most patients are males. ${ }^{2}$ The etiology and pathogenesis of LFH are not clearly defined. Thus, further studies and additional case reports will contribute to the present data. We report a case of LFH that occurred in association with spinal instability in a patient whose complaints were resolved rapidly following L5/S1 decompression surgery.

\section{Case Presentation}

A 60-year old man presented with a 3-week history of worsening low back pain radiating to the left leg. He had no recent history of trauma or lumbar surgery. His complaints were worsening during walking at approximately $50 \mathrm{~m}$. Neurological examination revealed left posterolateral leg pain on stretching and hypoesthesia on the left S1 dermatomes. In addition, straight leg raising test was positive on the left side.

(C)2020. Neurological Surgeons' Society of India.

This is an open access article published by Thieme under the terms of the Creative Commons Attribution-NonDerivative-NonCommercial-License, permitting copying and reproduction so long as the original work is given appropriate credit. Contents may not be used for commercial purposes, or adapted, remixed, transformed or built upon. (https://creativecommons.org/licenses/by-nc-nd/4.0/).

Thieme Medical and Scientific Publishers Pvt. Ltd. A-12, 2nd Floor, Sector 2, Noida-201301 UP, India 
His left Achilles reflex was hypoactive and there was also loss of left dorsiflexor muscle strength (3-4/5). Laboratory tests were unremarkable. Magnetic resonance imaging (MRI) without contrast was performed and revealed a left posterior epidural mass compressing the thecal sac at the left L5-S1 level. On the sagittal fat-suppressed T1-weighted image, there was a 1-cm hyperintense lesion that may be compatible with subacute stage hematoma (-Fig. 1A). On the T2-weighted images, the lesion was heterogeneously hypointense to spinal cord with hyperintense foci ( - Fig. 1B, C). Axial T2-weighted image showed compression of the thecal sac by the lesion at L5-S1 level (-Fig. 1C). Computed tomography (CT) images revealed the pars interarticularis defect at L5-S1 level ( - Fig. 2A, B). Surgical resection of the epidural lesion and transpedicular stabilization was recommended but the patient refused the application of instrumentation. Therefore, he underwent only left L5-S1 partial hemilaminectomy with excision of the epidural lesion under the aid of operation microscope. On early postoperative MRI, the lesion was seen as totally excised ( $\mathbf{- F i g . ~ 3 A , ~ B ) . ~ H e ~ r e c o v e r e d ~}$ rapidly after surgery with resolution of neurological symptoms and low back pain. Histopathological examination of the surgical specimen revealed presence of erythrocytes in ligamentous connective tissue and confirmed the diagnosis of LFH. There was no evidence of neural tissue or malignancy (-Fig. 4). When the blood biochemistry of the patient was examined retrospectively, no pathological finding that could cause bleeding diathesis were found.

\section{Discussion}

The LF is composed of elastic fibers (80\%) and collagen (20\%) and is poorly vascularized, having only a few small vessels pass through it in young healthy people. Therefore, intraligamentous bleeding is very rare. ${ }^{3-5}$ Although the pathogenesis of the development of hematoma is still unclear, it is thought to be a degenerative process. The most commonly accepted mechanism is the bleeding of the irregular small vessels of the degenerated and hypertrophied LF. ${ }^{6}$ Significant microangiogenesis around the ruptured collagen and elastic fibers during the degenerative changes in the LF has been reported. ${ }^{7}$ Epidemiological studies have showed the evidence of a causal role of mechanical stress due to spinal instability in initiating the pathological pathway of LF hypertrophy. ${ }^{8,9}$ In our patient, LFH was associated with the degenerated LF near the pars interarticularis defect at L5-S1 level. As a precipitating factor for hematoma, the most potential suspect is minor trauma resulting from daily activities or sports. In addition, percutaneous procedures like lumbar puncture or epidural interventions and increased intra-abdominal pressure are also accused for the tearing of these vessels. ${ }^{10}$ However, it may also occur as an idiopathic form without provacation. ${ }^{11,12}$ The clinical presentation of LFH is with radiculopathy or myelopathy according to the location of pathology, similar with the other more common causes of neural compression like disc herniation. When LFH occurs in the lumbar spine, the presentation is often with low back and/or leg pain accompanied by various neurological deficits as in our patient. LFH usually occurs acutely. Chronic LFH is rarely seen and a less common cause of severe neural compression. ${ }^{2}$ In the diagnosis, MRI is the most important tool for the detection of the relationship between the lesion in the epidural space and LF. ${ }^{1}$ In most LFH cases, lesions are hyperintense on T1-weighted MRIs, while heterogeneous intensity is seen on T2-weighted images. In our case, the LFH was also hyperintense on T1-weighted fat-suppressed MRIs that may be compatible with the subacute stage of hematoma. However, the intensities of the lesion can vary in relation to the stage of the hemoglobin breakdown during the aging of the blood. ${ }^{6}$ Therefore, it is sometimes very difficult to differentiate LFH from other epidural lesions like intraspinal cystic tumors or facet cysts. ${ }^{13}$ Contrast-enhanced MRI may help in the differentiation, because enhancement is much more rare in hematoma unlike spinal tumors. ${ }^{14}$ However, in LFH, slight-to-moderate peripheral enhancements may occur. ${ }^{15}$ In addition, there are reported cases of LFH with marked enhancement which
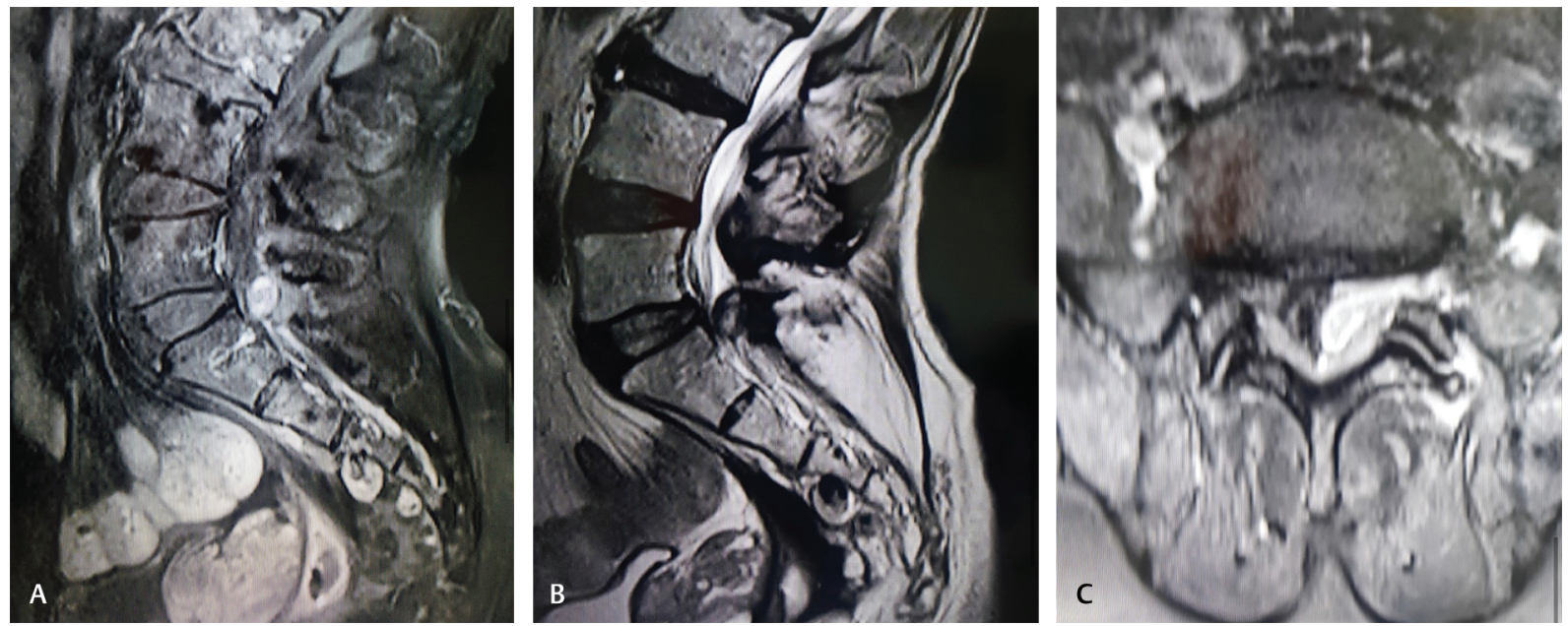

Fig. 1 Preoperative MRI of the lumbar spine without contrast showing left posterolateral epidural lesion, that is hyperintense on (A) sagittal T1-weighted MRI and heterogeneous on (B) sagittal T2-weighted MRI. This lesion exerts compression to the thecal sac at L5-S1 level on (C) axial T2-weighted MRI. MRI, magnetic resonance imaging. 

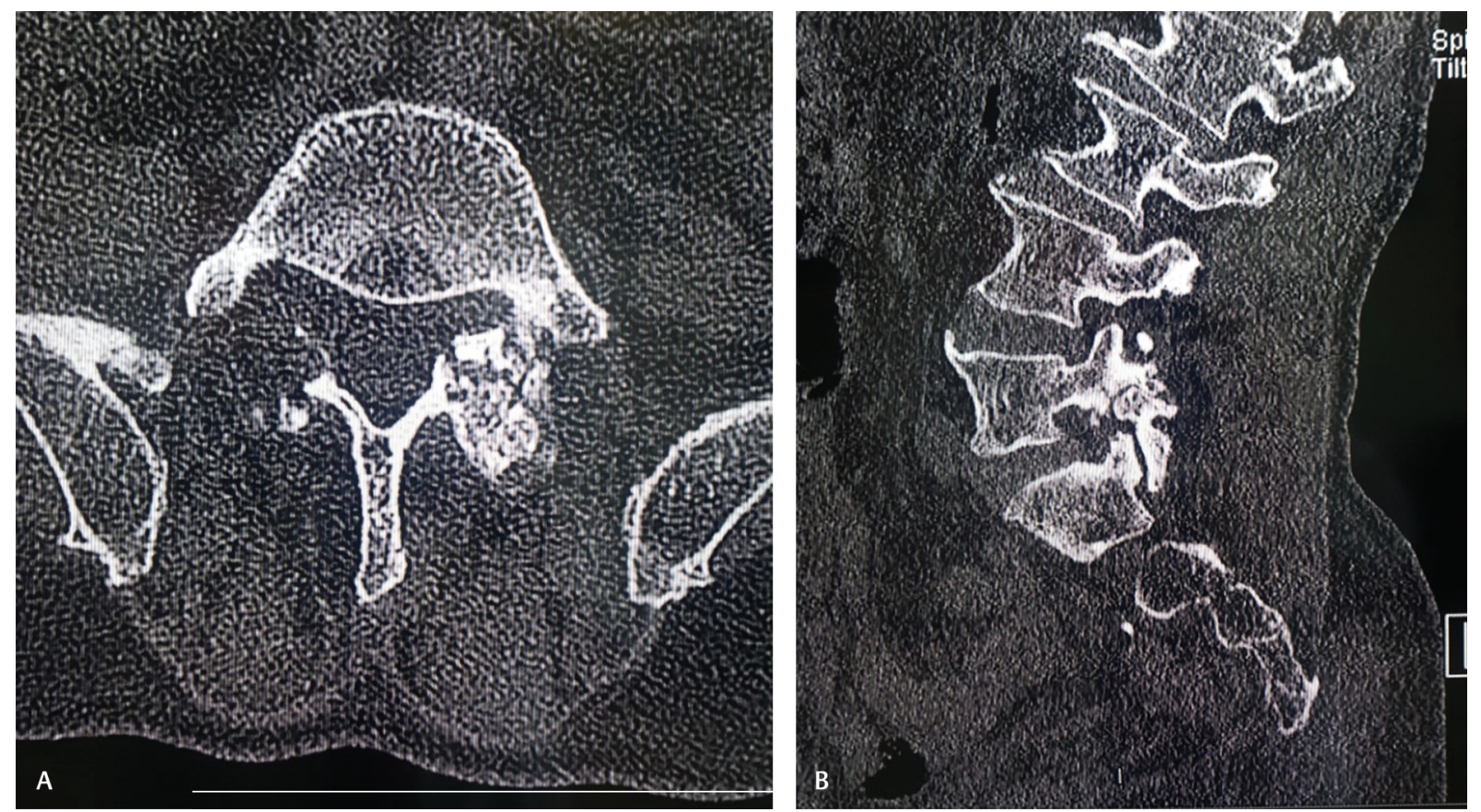

Fig. 2 Preoperative CT imaging of the lumbar spine showing the pars interarticularis defect at L5-S1 level on (A) the sagittal CT image and (B) the axial CT image. CT, computed tomography.
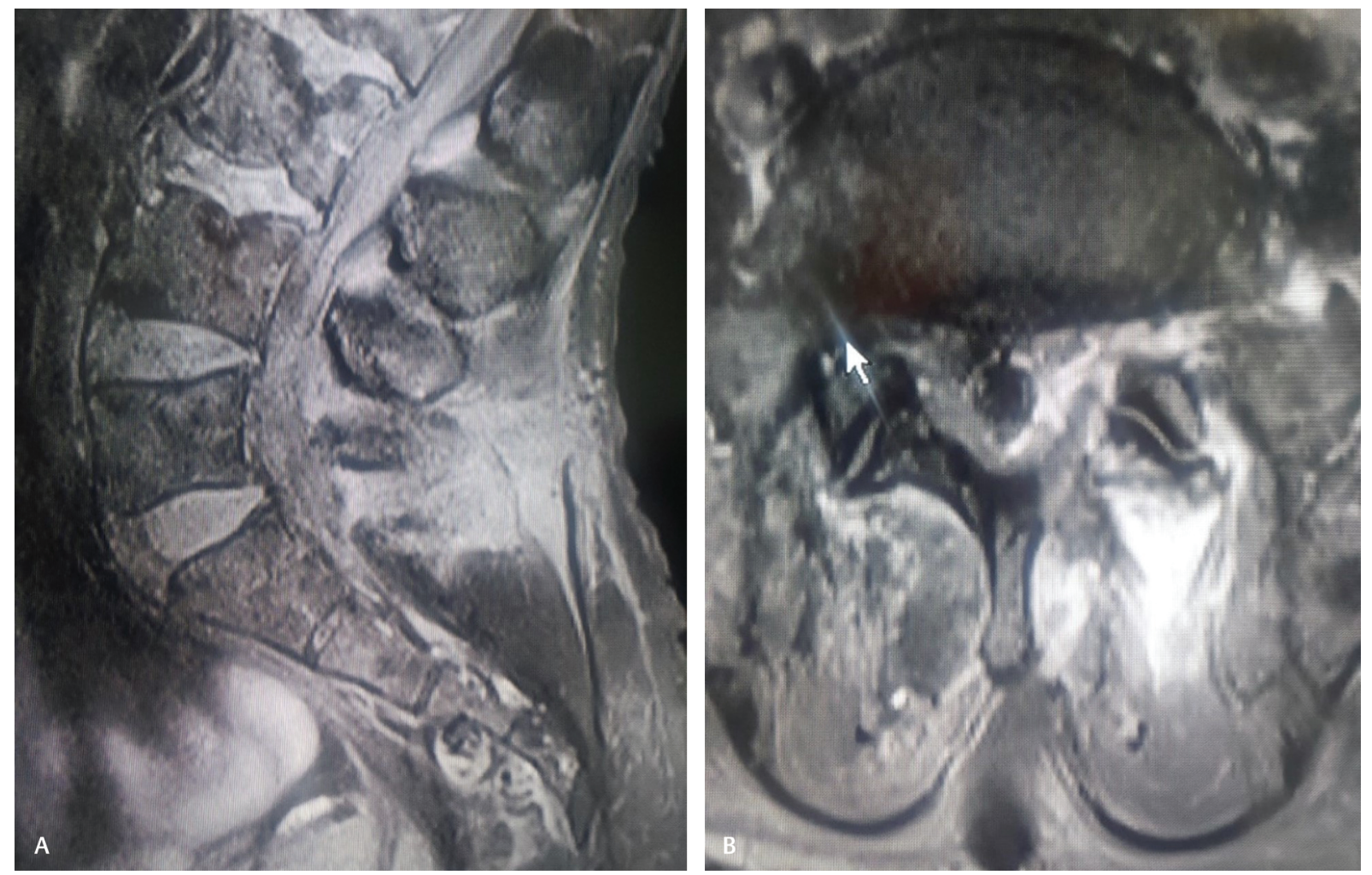

Fig. 3 Early postoperative MRI of the lumbar spine without contrast demonstrating the complete removal of the hematoma on (A) sagittal fat-suppressed T1-weighted MRI (B) axial T1-weighted MRI. MRI, magnetic resonance imaging.

causes preoperative suspicion that the lesion was likely to be neoplasm. ${ }^{16}$ Facet cysts are usually not enhanced, but they may also show rim enhancement. ${ }^{17}$ Consecutive MRIs may help the preoperative diagnosis by revealing signal intensity changes in relation to the stage of clothing. However, it may not be possible if the symptoms of the patient is severe. In patients who have severe pain and neurologic deficits, the standard care is surgical excision with rapid and favorable outcomes..$^{3,18,19}$ In our patient, we performed decompressive microsurgical excision of the lesion due to the presence of 


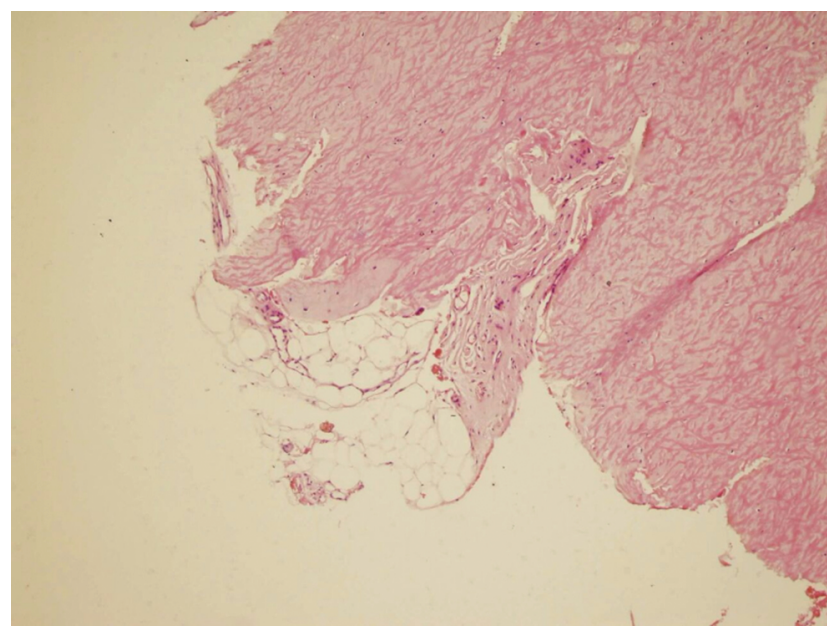

Fig. 4 Histological examination of the surgical specimen showing erythrocytes (red area) in ligamentous connective tissue (yellow area).

S1 radiculopathy. The prognosis of the patient after surgery was excellent. The diagnosis of LFH was made with the histopathological evaluation of the surgical specimen. Surgery is also recommended in some cases for the exact diagnosis. ${ }^{2}$ However, in stable patients, initial observation and conservative management may be warranted. ${ }^{20}$

\section{Conclusion}

LFH is an extremely rare cause of radiculopathy and/or myelopathy. However, it should be remembered in the differential diagnosis of the epidural lesions, especially when it is seen in the lumbar region in older-aged male patients. Spinal instability that plays a role in the pathogenesis of hypertrophy and degeneration of LF is one of the cause of LFH. The patients who have severe pain and neurological deficit should be surgically managed immediately.

\section{Conflict of Interest}

None declared.

\section{References}

1 Sweasey TA, Coester HC, Rawal H, Blaivas M, McGillicuddy JE. Ligamentum flavum hematoma. Report of two cases. J Neurosurg 1992;76(3):534-537

2 Shafaq N, Suzuki A, Terai H, Wakitani S, Nakamura H. Cellularity and cartilage matrix increased in hypertrophied ligamentum flavum: histopathological analysis focusing on the mechanical stress and bone morphogenetic protein signaling. J Spinal Disord Tech 2012;25(2):107-115

3 Kono H, Nakamura H, Seki M, Motoda T. Foot drop of sudden onset caused by acute hematoma in the lumbar ligamentum flavum: a case report and review of the literature. Spine 2008;33(16):E573-E575
4 Mizuno J, Nakagawa $\mathrm{H}$, Inoue $\mathrm{T}$, Hashizume Y. Ligamentum flavum hematoma in the lumbar spine. Neurol Med Chir (Tokyo) 2005;45(4):212-215

5 Kosaka H, Sairyo K, Biyani A, et al. Pathomechanism of loss of elasticity and hypertrophy of lumbar ligamentum flavum in elderly patients with lumbar spinal canal stenosis. Spine 2007;32(25):2805-2811

6 Cui G, Watanabe K, Miyauchi Y, et al. Matrix metalloproteinase 13 in the ligamentum flavum from lumbar spinal canal stenosis patients with and without diabetes mellitus. J Orthop Sci 2011;16(6):785-790

7 Yayama T, Kobayashi S, Sato R, et al. Calcium pyrophosphate crystal deposition in the ligamentum flavum of degenerated lumbar spine: histopathological and immunohistological findings. Clin Rheumatol 2008;27(5):597-604

8 Hur JW, Kim BJ, Park JH, et al. The mechanism of ligamentum flavum hypertrophy: introducing angiogenesis as a critical link that couples mechanical stress and hypertrophy. Neurosurgery 2015;77(2):274-281, discussion 281-282

9 Sairyo K, Biyani A, Goel V, et al. Pathomechanism of ligamentum flavum hypertrophy: a multidisciplinary investigation based on clinical, biomechanical, histologic, and biologic assessments. Spine 2005;30(23):2649-2656

10 Kotil K. Severe neurologic deficit caused by chronic ligamentum flavum hematoma: the first case series. J Neurol Sci 2015;32:391-398

11 Eltorai AEM, Kuris EO, Lucas PR, Daniels AH. Idiopathic ligamentum flavum hematoma. Spine J 2016;16(5):e307

12 Keynan O, Smorgick Y, Schwartz AJ, Ashkenazi E, Floman Y. Spontaneous ligamentum flavum hematoma in the lumbar spine. Skeletal Radiol 2006;35(9):687-689

13 Salehpour F, Mirzaei F, Rezakhah A, Aeinfar K, Kazemzadeh M, Alavi SAN. Ligamentum flavum hematoma presented with low back pain: a case report and review of the literature. Int J Spine Surg 2018;12(3):337-341

14 Kotani Y, Sudo H, Abumi K, Ito M, Matsubara S, Minami A. Spinal cord compression by hematoma in the cervical ligamentum flavum: a case report. Spine J 2012;12(4):e18-e21

15 Takahashi M, Satomi K, Hasegawa A, Hasegawa M, Taki N, Ichimura S. Ligamentum flavum hematoma in the lumbar spine. J Orthop Sci 2012;17(3):308-312

16 Ghent F, Ye X, Yan M, Mobbs RJ. A contrast-enhancing lumbar ligamentum flavum haematoma. BMJ Case Rep 2014;2014:bcr2013202521

17 Park JB, Kong CG, Suhl KH, Chang ED, Riew KD. The increased expression of matrix metalloproteinases associated with elastin degradation and fibrosis of the ligamentum flavum in patients with lumbar spinal stenosis. Clin Orthop Surg 2009;1(2):81-89

18 Lakemeier S, Schmid R, Foltz L, et al. Increased expression of CD44 in hypertrophied ligamentum flavum and relevance of splice variants CD44v5 and CD44v6. Acta Neurochir (Wien) 2012;154(2):359-365, discussion 365

19 Yüceer N, Başkaya MK, Smith P, Willis BK. Hematoma of the ligamentum flavum in the lumbar spine: case report. Surg Neurol 2000;53(6):598-600

20 Algattas H, Kimmell KT, Petraglia AL, Maurer PK. Conservative management of a cervical ligamentum flavum hematoma: case report. Surg Neurol Int 2016;7(suppl 3) :S61-S63 